\title{
A message for 2018
}

\author{
Naomichi Matsumoto Editor-in-Chief ${ }^{1,2}$
}

Received: 21 January 2018 / Accepted: 21 January 2018 / Published online: 26 March 2018

(c) The Author(s) under exclusive licence to The Japan Society of Human Genetics 2018

The Journal of Human Genetics published 169 papers in 2017. We prepared a special issue describing "genome editing" in 2017, which was published in February 2018. I would like to express my appreciation to guest editors, Drs. Tetsushi Sakuma and Takashi Yamamoto, and all the contributors for their hard work. I hope the special issue will attract attention from a broad readership.

In 2018, we are again planning a special issue. I am looking forward to it, and of course to receiving more

Jun Abe

Margaret Adam

David Adams

Masato Akiyama

Hasan Akman

Hidenori Akutsu

Anas Alazami

Enrico Alfei

Salam Alkindi

Fowzan Alkuraya

Folefac Aminkeng

Anthony Antonellis

Yoko Aoki

Yoshitsugu Aoki

Walter Arancio

Takahiro Arima

Takuro Arimura

Hiroyuki Awano

Qasim Ayub

Anitha Ayyappan Pillai

Noriyuki Azuma

Claire Bellis
Tsuyoshi Baba
Patricia Balaresque
Doron Behar
Jin-xin Bei
Robert Bennett
Minako Beppu
Christophe Beroud
Hemant Kumar Bid
Abigail W Bigham
Gerhard Binder
Ohad Birk
Johannes Birtel
Lorenzo Botto
Michael Brigg
Maria Brion
Carolyn Brown
Susan Brown
Ramon Brugada
Tom Brutseart
Jane Burns

Claire Bellis

Tsuyoshi Baba

Doron Behar

Jin-xin Bei

Robert Bennett

Minako Beppu

Christophe Beroud

Hemant Kumar Bid

Abigail W Bigham

Gerhard Binder

Ohad Birk

Johannes Birtel

Lorenzo Botto

Michael Brigg

Maria Brion

Carolyn Brown

Susan Brown

Tom Brutseart

Jane Burns exciting manuscripts for the Journal of Human Genetics from all over the world.

Acknowledgements The Editor-in-Chief, on behalf of the Journal of Human Genetics Editorial Board and the Japan Society of Human Genetics, gratefully acknowledges the generous support from peer reviewers in carefully assessing manuscripts under consideration. The following individuals provided reviews of manuscripts submitted to the Journal of Human Genetics in 2017:

\author{
Neal Copeland \\ Daniel Corach \\ Carolyn Coulam \\ John Creemers \\ John Dagl \\ Coleen Damcott \\ Marion Danner \\ Sumito Dateki \\ Mehul Dattani \\ Ronny Decorte \\ Demetrio Domingo \\ Rajkumar Dorajoo \\ Debra Doyle \\ David Duffy \\ Ana Duggan \\ Fumio Endo \\ Williams C Earnshaw \\ Yusuke Ebana \\ Ken Ebihara \\ Alexis C. Edwards \\ Thomas Eggermann
}

Naomichi Matsumoto

naomat@yokohama-cu.ac.jp

Journal of Human Genetics

2 Department of Human Genetics, Yokohama City University Graduate School of Medicine, Yokohama, Japan 


\begin{tabular}{|c|c|}
\hline Mariko Eguchi & Kenichiro Hata \\
\hline John Eicher & Izuho Hatada \\
\hline Dr. Syed Hassan Ejaz Zaidi & Naoko Hattori \\
\hline Ayman El-Hattab & Hisao Hayashi \\
\hline John A. Elefteriades & Kenshi Hayashi \\
\hline Alison Elliott & Shin Hayashi \\
\hline Masatsugu Ema & Takaaki Hayashi \\
\hline Beverly Emanuel & Takeharu Hayashi \\
\hline Wolfgang Enard & Yukiko Hayashi \\
\hline Andrew Engel & Karen Heath \\
\hline Vermeulen Eric & Robert Hegele \\
\hline A Fahim & Sarah Heron \\
\hline Huitao Fan & Christin Hertz \\
\hline Audrey Farrugia & Evelyne Heyer \\
\hline Alessandra Ferlini & Tetsuya Hirata \\
\hline Roslyn Fitch & Kouyuki Hirayasu \\
\hline Kevin Flanigan & Shinichi Hirose \\
\hline Jia Nee Foo & Tomomitsu Hirota \\
\hline Jay Fowke & Yuki Hitomi \\
\hline Nelson Freimer & Edward Hollox \\
\hline Kathryn French & Kohei Homma \\
\hline Katsunori Fujii & Yukio Horikawa \\
\hline Akihiro Fujimoto & Satoyo Hosono \\
\hline Ikuma Fujiwara & Akitsu Hotta \\
\hline Maki Fukami & Miles Houslay \\
\hline Koya Fukunaga & Timothy Howard \\
\hline Akimune Fukushima & Katherine Howell \\
\hline Tod Fullston & Ching-Sheng Hsu \\
\hline Hiroshi Furukawa & Martijn Huynen \\
\hline Bo Gao & Tomohiko Ichikawa \\
\hline Angelina García & Shingo Ichimiya \\
\hline Leslie Gordon & Masahiro Iijima \\
\hline Jun Goto & Kazuyuki Ikeda \\
\hline Yu-ichi Goto & Masashi Ikeda \\
\hline Yusuke Goto & Minako Imamura \\
\hline Anne Greenough & Issei Imoto \\
\hline Giedre Grigelioniene & Hidehito Inagaki \\
\hline Markus Grompe & Akihito Inoko \\
\hline Tomasz Grzybowski & Hidetoshi Inoko \\
\hline Francesca Gualandi & Jun Inoue \\
\hline Elizabeth Guerrero-Berroa & Astrid Irwanto \\
\hline Philip Guthrie & Isao Ishida \\
\hline TomonobuHasegawa & Takafumi Ishida \\
\hline Nobuhiko Haga & Kazuyoshi Ishigaki \\
\hline Kazuhiro Haginoya & Tetsuya Ishii \\
\hline Hiromi Hamada & Kinya Ishikawa \\
\hline Hiromichi Hamada & Taisuke Ishikawa \\
\hline Kazushige Hanaoka & Kenji Ishikura \\
\hline Yangyang Hao & Hiroyuki Ishiura \\
\hline Masaru Harada & Minoru Isomura \\
\hline Naoki Harada & Hidemi Ito \\
\hline Koshi Hashimoto & Kaoru Ito \\
\hline Naoki Hashizume & Shuichi Ito \\
\hline
\end{tabular}

Tetsuya Ito

Kazuya Iwamoto

Satoshi Iwasaki

Kosuke Izumi

Emmanuel Jacquemin

Aashish Jha

Timothy Jinam

Colin Johnson

Michael Köttgen

Hiroshi Kawame

Frank J Kaiser

Marko Johannes Kallio

Yoichiro Kamatani

YasmineKanaan

Tadashi Kaname

Tetsufumi Kanazawa

Peter Kannu

Piranit Kantaputra

Mureo Kasahara

Naoyuki Kataoka

Kazuto Kato

Mitsuhiro Kato

Takema Kato

Aya Kawasaki

Haig H. Kazazian

Naoto Keicho

Janet Kelso

Kim Keppler-Noreuil

Christine Keyser

Atsuo Kikuchi

Takashi Kikuiri

Jung Wook Kim

Sung-Won Kim

En Kimura

Masaki Kimura

Tadashi Kimura

Takashi Kinoshita

Ryutaro Kira

Noriyuki Kishi

Priya Kishnani

Hiroshi Kitoh

Toomas Kivisild

Kazuma Kiyotani

Jürgen Klammt

Anita Kloss-Brandstätter

Masahisa Kobayashi

Yuta Kochi

Hiroko Kodama

Tsuyoshi Koide

Akio Koizumi

Satoko Kojima

Shinji Kondo

Tatsuro Kondoh
Genevieve Konopka

Kenjiro Kosaki

Rika Kosaki

Tomoki Kosho

Manoelle Kossorotoff

Motomichi Kosuga

Zsofia Kote-Jaraj

Toru Kubo

Mitsuru Kubota

TaKeo Kubota

Tomu Kuchikata

Taco Kuijpers

Jerzy Kulski

Takayoshi Kuno

Hiroki Kurahashi

Shigeo Kure

Yoko Kuroki

Kenji Kurosawa

Sasagu Kurozumi

Wibhu Kutanan

Kerstin Kutsche

Maarten Larmuseau

Chris Lau

Yu-Lung Lau

Hwan Young Lee

Tomoko Lee

Eric Legius

Rachel Lennon

Hui Li

Jingmei Li

Yumei Li

Andrew Lidral

Hsi-Hsien Lin

Annika Lindblom

Hong Liu

BastienLlamas

Chengzu Long

Siew-Kee Low

Michelle Luciano

Friedrich Luft

Donata Luiselli

Artem Lysenko

Yoshio Makita

Shinsei Minoshima

Outi Mäkitie

Lionel Maldergem

Boris Malyarchuk

Elisabeth Mangold

Shuhei Mano

Teri Manolio

Nina Marchi

Tomislav Maricic

Arseni Markoff 
Deborah Marshall

Marcella Martinelli

Osamu Masui

Hironori Masuko

Koichi Matsuda

Takayoshi Matsumura

Tatsuo Matsunaga

Hirotaka Matsuo

Masafumi Matsuo

Tohru Matsuura

Takashi Matsuzaka

Hamdi Mbarek

Steven McCarroll

Donna M. McDonald-

McGinn

Eckart Meese

Fanny Mendisco

Luciano Merlini

Tony R Merriman

Mait Metspalu

Innes Micheil

Daiki Miki

Dianna Milewicz

Francisca Millan

Jeff Milunsky

Kazuharu Misawa

Sonoko Misawa

Hiroyuki Mishima

Norisato Mitsutake

Kiyonori Miura

Fuyuki Miya

Hidehiko Miyake

Noriko Miyake

Toshinobu Miyamoto

Satoko Miyatake

Satoru Miyawaki

Rikke Moller

Naoya Morisada

Hiroyuki Morita

Bernice E Morrow

Celia Moss

Stefan Mundlos

Masaaki Muramatsu

Kei Murayama

Koji Muroya

Taisei Mushiroda

Mathias Muszlak

Kaori Muto

Kenji Naritomi

Gen Nishimura

Hiranao Numabe

Koji Nagao

Hiroaki Nagase
Ali Naini

Kazuhiko Nakabayashi

Masanori Nakagawa

Yoko Nakajima

Akinori Nakamura

Kimitoshi Nakamura

Makoto Nakamura

Hirofumi Nakaoka

Hiromitsu Nakauchi

Kazuhiro Nakayama

Tomohiro Nakayama

Eiji Nanba

Sergey Nejentsev

Marcella Neri

Kypros H Nicolaides

Gael Nicolas

Vincenzo Nigro

Nao Nishida

Chikako Nishigori

Fumichika Nishimura

Hisahide Nishio

Shinya Nishio

Emiko Noguchi

Hisashi Noma

Luigi Notarangelo

Michael Nothnagel

Shusuke Numata

William Nyhan

Hirofumi Ohashi

TorayukiOkuyama

Chikashi Obuse

Katsuhisa Ogata

Tsutomu Ogata

Masanobu Ogawa

Yoshihiro Ogawa

Yuya Ogawa

Jun Ohashi

Toya Ohashi

Tatsuya Ohhata

Seiko Ohno

Tohru Ohta

Akira Ohtake

Toshihiro Ohura

Kimihiko Oishi

Yukinori Okada

Nobuhiko Okamoto

Yasushi Okazaki

Osamu Onodera

Yoshihiro Onouchi

Raffaella Origa

Hitoshi Osaka

Takahiro Otani

Takeshi Otowa
Jurg Ott

Outi Outi Mäkitie

Kouichi Ozaki

Yasuhiko Ozaki

Nobuaki Ozawa

Takeshi Ozeki

Luisa Politano

Brigitte Pakendorf

Terry Partridge

Eric Pasmant

Ashwini Patil

Min-Sheng Peng

Luisa Pereira

Aline Petrin

Frédéric B.Piel

Y Pommier

Josef Prchal

Irina Pugach

Xuebin Qi

Paula Ramos

Gudrun Rappold

Wendy Raskind

Pierre F Ray

Marcelo Ribeiro

ÂndreaRibeiro-dos-Santos

François-XavierRicaut

Martin Richards

Paola Rimessi

Merryl Rodrigues

Lutz Roewer

Urszula Rogalla

Noah Rosenberg

Guy Rouleau

Deborah Rund

Nobuyuki Shimozawa

Shigeaki Sunada

Rani Sachdev

Lynette Sadleir

Takashi Sado

Vladimir Saenko

Shigeru Saito

Yoshiaki Saito

Shinji Saitoh

Hirotomo Saitsu

Aiko Sakai

Norio Sakai

Yasunari Sakai

Osamu Sakamoto

Tetsushi Sakuma

Akihiro Sakurai

Antonio Salas

Osamu Samura

Junji Saruwatari
Yasushi Sasaki

Toshikuni Sasaoka

Takehiro Sato

Takeshi Sato

Robert Saul

Hiromi Sawai

Sebastian Schönherr

Johanna Schleutker

Brian Schutte

Daryl Scott

Naohiko Seki

Kenji Sekiguchi

Akihiko Sekizawa

Marco Seri

Jun Sese

Alok Sharma

A. Eliot Shearer

Volney Sheen

Saumya Shekhar

Ying Shen

Stephen Sherry

Daichi Shigemizu

Takashi Shiina

Haruo Shimazaki

Atsushi Shimizu

Kenji Shimizu

Norikazu Shimizu

Keiko Shimojima

Kyoung-Jin Shin

Koji Shiraishi

Kouya Shiraishi

Yuichi Shiraishi

Vorasuk Shotelersuk

Xueling Sim

Katherine A. Siminovitch

Tatum S. Simonson

Karl Skorecki

Brian Skotko

Anne Slavotinek

Jana Slyskova

Richard Smith

Pedro Soares

Hidenobu Soejima

Jun Sone

Shinichi Sonta

Ulrich Specks

Martin H Steinberg

Mary Stephenson

David Stevenson

Mark Stoneking

Lisa Strain

James Stull

Mayumi Sugiura-Ogasawara 
Chonlaphat Sukasem

Bhoom Suktitipat

Andrea Superti-Furga

Kanya Suphapeetiporn

Akari Suzuki

Carolyn K Suzuki

Hideo Suzuki

Motoshi Suzuki

Satoshi Suzuki

Teruhiko Suzuki

Yoichi Suzuki

Katarzyna Szymanska

Hayato Tada

Masataka Taguri

Atsushi Tajima

Go Tajima

Toshihiro Tajima

Shuji Takada

Atsushi Takahashi

Yuji Takahashi

Kyoko Takano

Miho Takaoka

Hiroshi Takashima

Takanori Takebe

Junji Takeda

Shin'ichi Takeda

Toshiki Takenouchi

Fumihiko Takeuchi

Takashi Taki
Gen Tamiya

Chieko Tamura

Tiong Tan

Noriko Tanabe

Toshihiro Tanaka

Yoko Tanaka

Nelson L.S. Tang

Tang K Tang

Atsuo Taniguchi

Mariko Taniguchi-Ikeda

Chizu Tanikawa

Kenji Tanimura

Lorena Tapia

Jack Tarleton

Chikashi Terao

Giovanni Tiscia

Micheal To

Takao Togawa

Katsushi Tokunaga

Shunji Tomatsu

Hidefumi Tonoki

Ulises Toscanini

Shih-Feng Tsai

Koichiro Tsugawa

Henna Tyynismaa

Takehiro Ueda

Mayumi Ueta

Guy Van Camp

Johan Van de Walle
Ingrid Van de laar

Adeline Vanderver

Sonja Vernes

Dario Vianello

Alexandre Vieira

David Viskochil

Pavel Vodicka

Peter Vogt

Keiko Wakui

Takahito Wada

Tom Walsh

Chuan-Chao Wang

Dong Wang

Fan Wang

Matthew Warman

Atsushi Watanabe

Mari Wataya-Kaneda

Hansi Weissensteiner

Kevin P. White

Matthew Wicklund

Fredrik Wiklund

Marc Willinger

Timothy Wright

Hui Xiong

Shuhua Xu

Yali Xue

Ichiro Yabe

Nami Yamada

Ryo Yamada
Yasukazu Yamada

Midori Yamamoto

Toshiyuki Yamamoto

Kenji Yamashiro

Keiko Yamazaki

Kazuki Yamazawa

Kunimasa Yan

Hideki Yanai

Wanling Yang

Ichiro Yokota

Han-Wook Yoo

Tohru Yorifuji

Kunihiro Yoshida

Hiroshi Yoshihashi

Kazuto Yoshimi

Tsuneaki Yoshinaga

Koh-ichiro Yoshiura

Kosuke Yusa

Andreas Zankl

Changqing Zeng

Massimo Zeviani

Tongwen Zhang

Xiaoming Zhang

Xue-jun Zhang

Yangbing Zhao

Zhi-Gang Zhao

Pingping Zheng

Qing Zhou

Marcella Zollino 